\title{
Study on Vibration Characteristics on Directionally Solidified Blade*
}

\author{
Yasutomo KANEKO** \\ ** Department of Mechanical and Systems Engineering, Ryukoku University \\ 1-5 Yokotani Oe-Cho Seta Otsu, Siga, Japan \\ E-mail: y_kaneko@rins.ryukoku.ac.jp
}

\begin{abstract}
Recently, DS (Directionally Solidified) and SC (Single Crystal) alloys have been widely applied for gas turbine blades instead of CC (Conventionally Casting) alloys to meet the requirement of the high temperature operation and to improve the thermal efficiency. The SC blade consists of one columnar grain, and the DS blade consists of several columnar grains where the growing direction of the columnar crystal is set to the direction of the centrifugal force. Therefore, the vibration analysis of the SC and DS blades has to be carried out, taking account of the anisotropy. In this study, first, the effect of the anisotropy of elastic constants on the vibration characteristics of the SC and DS blades are investigated in detail. Second, the validity of the assumption of the transverse isotropy for the DS blade is examined, carrying out the Monte Carlo simulation. From these results, it is concluded that the actual DS blade can be treated as a transversely isotropic material.
\end{abstract}

Key words: Turbomachinery, Vibration of Rotating Body, Blade, Resonance

\section{Introduction}

Recently, DS (Directionally Solidified) and SC (Single Crystal) alloys have been widely applied for gas turbine blades instead of CC (Conventionally Casting) alloys to meet the requirement of the high temperature operation and to improve the thermal efficiency ${ }^{(1)(2)}$. The SC blade consists of one columnar grain, and therefore the creep rupture and thermal fatigue resistance can be improved by eliminating the grain boundary. Since the elastic constants of the SC blades are anisotropic, the vibration analysis of the SC blade has to be carried out, taking account of the anisotropy. On the other hand, the DS blade consists of several columnar grains where the growing direction of the columnar crystal is set to the direction of the centrifugal force to yield a large creep resistance by eliminating the grain boundary perpendicular to the loading direction. The vibration analysis of the DS blade also has to be carried out, taking account of the anisotropy because the direction of the columnar crystal growth is parallel to the loading direction.

In the vibration analysis of the DS blade by FEA (Finite Element Analysis), it seems that all of the columnar grains included in the blade have to be modeled exactly ${ }^{(3)(4)}$. In practice, however, it is difficult to know the exact growing direction and the geometry of the columnar grain. It is reported that if the volume and the rotation angle in the transverse plane of each columnar crystal are known, the vibration analysis of the DS blade can be carried out with the practical accuracy by use of the elastic constants averaged by the Reuss and Voigt method ${ }^{(5)}$. However, it is also difficult to get such data of the actual DS blade in advance. Therefore, in the actual mechanical design of the DS blade, the vibration analysis is carried out, assuming that the number of the columnar grains included in the blade is 
large, and the DS blade can be considered transverse isotropic ${ }^{(1)}$. In this study, taking account of the above-mentioned situation, first, the effect of the anisotropy of elastic constants on the vibration characteristics of the SC and DS blade is investigated. Second, the validity of the assumption of the transverse isotropy for the DS blade is examined, carrying out the Monte Carlo simulation. That is, in the actual DS blade, the number of the columnar grains included in the blade is 5 to 10 at most, and is not enough. Therefore, whether the assumption of the transverse isotropy can be applied to the vibration analysis of the actual DS blade is examined.

\section{Analysis Method}

\subsection{Elastic Stress-Strain Relationship of SC and DS Blade ${ }^{(1)}$}

This paper employs two coordinate systems as shown in Fig.1 and Fig. 2. One is the structural coordinate expressed by the small letter $x_{1}, x_{2}$, and $x_{3}$, which defines the geometry of the blade. The other system is the alloy coordinate system, expressed by the capital letter $X_{1}, X_{2}$, and $X_{3}$ and fixed to each columnar grain. The alloy coordinate system are directed to the crystal axes of [100], [010], and [001]. The angle $\theta$ defines the rotation angle around [010] axis, while the angle $\phi$ defines the rotation angle around [001] axis. The angle $\gamma$ defines the rotation angle around [100] axis, which is not written in Fig. 2 for lack of space.

In general, the elastic stress strain relationship (generalized Hooke's law) for the orthotropic materials can be expressed by Eq.(1), taking the coordinate along the crystal axis as shown in Fig. $1^{(1)}$.

$$
\left\{\begin{array}{l}
\varepsilon_{11} \\
\varepsilon_{22} \\
\varepsilon_{33} \\
\gamma_{12} \\
\gamma_{23} \\
\gamma_{31}
\end{array}\right\}=\left[\begin{array}{cccccc}
S_{11} & S_{12} & S_{13} & 0 & 0 & 0 \\
S_{12} & S_{22} & S_{23} & 0 & 0 & 0 \\
S_{13} & S_{23} & S_{33} & 0 & 0 & 0 \\
0 & 0 & 0 & S_{44} & 0 & 0 \\
0 & 0 & 0 & 0 & S_{55} & 0 \\
0 & 0 & 0 & 0 & 0 & S_{66}
\end{array}\right]\left\{\begin{array}{l}
\sigma_{11} \\
\sigma_{22} \\
\sigma_{33} \\
\tau_{12} \\
\tau_{23} \\
\tau_{31}
\end{array}\right\}
$$

Where, $S_{i j}$ is the element of the compliance matrix $[S]$ in the $X_{1} X_{2} X_{3}$ coordinate, $\sigma_{i j}$ the normal stress component, $\tau_{i j}$ the shear stress component, $\varepsilon_{i j}$ the normal strain component, $\gamma_{i j}$ the shear strain component, respectively.

Nickel base alloys are used as the material of the SC and DS blade of gas turbine. They are the face centered cubic, and the elastic stress strain relationship in the single crystal alloy can be expressed by Eq.(2), because the material characteristics are symmetric with respect to $X_{1}, X_{2}$, and $X_{3}$ axis.

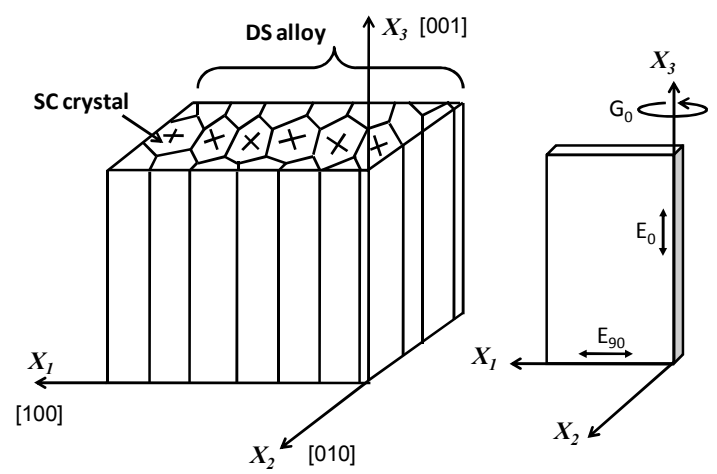

Fig. 1 Coordinate and material property of SC and DS alloy 


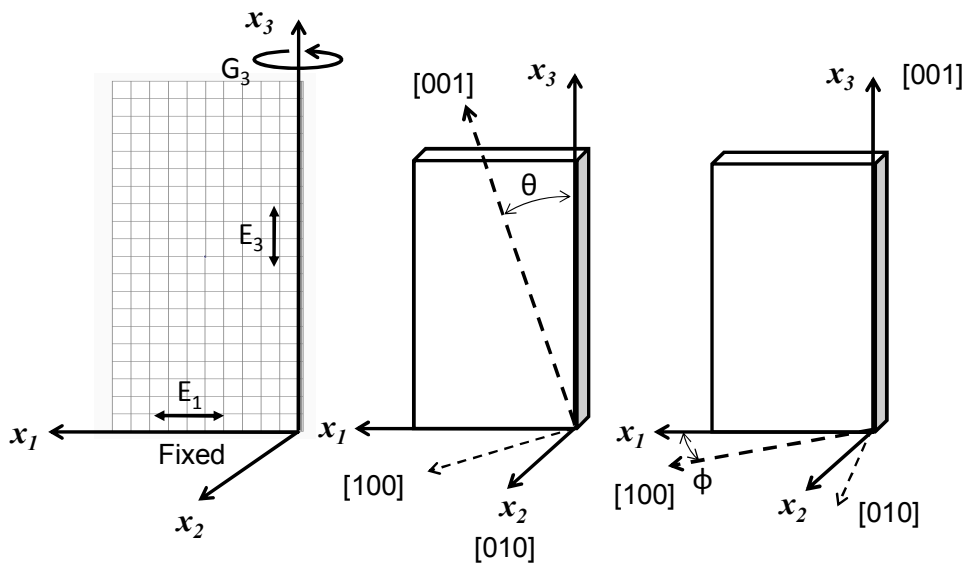

Fig. 2 Coordinate system of blade and FE model

$$
\left\{\begin{array}{l}
\varepsilon_{11} \\
\varepsilon_{22} \\
\varepsilon_{33} \\
\gamma_{12} \\
\gamma_{23} \\
\gamma_{31}
\end{array}\right\}=\left[\begin{array}{cccccc}
S_{33} & S_{13} & S_{13} & 0 & 0 & 0 \\
S_{13} & S_{33} & S_{13} & 0 & 0 & 0 \\
S_{13} & S_{13} & S_{33} & 0 & 0 & 0 \\
0 & 0 & 0 & S_{44} & 0 & 0 \\
0 & 0 & 0 & 0 & S_{44} & 0 \\
0 & 0 & 0 & 0 & 0 & S_{44}
\end{array}\right]\left\{\begin{array}{c}
\sigma_{11} \\
\sigma_{22} \\
\sigma_{33} \\
\tau_{12} \\
\tau_{23} \\
\tau_{31}
\end{array}\right\}
$$

In the single crystal alloy, the independent constants are three of $S_{33}, S_{13}$ and $S_{44}$, which can be expressed by Eq.(3), using the engineering elastic constants.

$$
S_{33}=1 / E_{0}, \quad S_{13}=-v_{0} / E_{0}, \quad S_{44}=1 / G_{0}
$$

Where, $E_{0}$ is Young's modulus, $G_{0}$ the shear rigidity, $v_{0}$ Poisson's ratio with respect to $X_{3}$ axis. In Eq.(3), the subscript ' 0 ' denotes the quantity corresponding to the $X_{3}$ axis as shown in Fig. 1. If the material is isotropic, Eq.(4) is satisfied. Therefore, the independent material constants are two of $E_{0}$ and $G_{0}$.

$$
G_{0}=\frac{E_{0}}{2\left(1+v_{0}\right)}
$$

The DS alloy is a bundle of single crystals having the same $X_{3}$ direction (the solidification direction) as shown in Fig. 1. In other words, although the solidification directions of all single crystals in the DS alloy are the same, the crystal direction in the $X_{1}-X_{2}$ plane is random. Therefore, if the number of the single crystals is large, the material property in this plane can be treated isotropically, and the stress strain relationship of the DS alloy can be expressed by Eq.(5).

$$
\left\{\begin{array}{l}
\varepsilon_{11} \\
\varepsilon_{22} \\
\varepsilon_{33} \\
\gamma_{12} \\
\gamma_{23} \\
\gamma_{31}
\end{array}\right\}=\left[\begin{array}{cccccc}
\overline{S_{11}} & \overline{S_{12}} & \overline{S_{13}} & 0 & 0 & 0 \\
\overline{S_{12}} & \overline{S_{11}} & \overline{S_{13}} & 0 & 0 & 0 \\
\overline{S_{13}} & \overline{S_{13}} & \overline{S_{33}} & 0 & 0 & 0 \\
0 & 0 & 0 & \overline{S_{44}} & 0 & 0 \\
0 & 0 & 0 & 0 & \overline{S_{55}} & 0 \\
0 & 0 & 0 & 0 & 0 & \overline{S_{55}}
\end{array}\right]\left\{\begin{array}{c}
\sigma_{11} \\
\sigma_{22} \\
\sigma_{33} \\
\tau_{12} \\
\tau_{23} \\
\tau_{31}
\end{array}\right\}
$$


Where, $\overline{S_{i j}}$ denotes the element of the compliance matrix for the DS alloy which is assumed to be isotropic in $X_{1}-X_{2}$ plane, and the Eq.(6) is satisfied.

$$
\overline{S_{44}}=2\left(\overline{S_{11}}-\overline{S_{12}}\right)
$$

Since the material constants in $X_{3}$ axis is the same as the single crystal, Eq.(7) is derived. In this case, independent material constants are five.

$$
\overline{S_{33}}=S_{33}, \overline{S_{55}}=\overline{S_{66}}=S_{44}, \overline{S_{13}}=\overline{S_{23}}=S_{13}
$$

Material constants of the DS alloy in the $X_{1}-X_{2}$ plane can be obtained, averaging the material constants of the SC alloy in the transverse plane under the assumption that the distribution of the crystal direction in the plane is random. The material constants of the DS alloy in the $X_{1}-X_{2}$ plane can be described by Eq.(8), if the Wells' method ${ }^{(6)}$ is adopted in averaging.

$$
\begin{aligned}
& \overline{S_{11}}=\overline{S_{22}}=\frac{1}{2} \sqrt{S_{33}\left(2 S_{33}+2 S_{13}+S_{44}\right)}, \\
& \overline{S_{44}}=\sqrt{2 S_{44}\left(S_{33}-S_{13}\right)}
\end{aligned}
$$

And Eq.(9) is also derived from Eq.(6).

$$
\overline{S_{12}}=\overline{S_{11}}-\frac{\overline{S_{44}}}{2}
$$

In short, if the number of the single crystal alloys is large enough to assume that the material is isotopic in the $X_{1}-X_{2}$ plane, the independent material constants become three. Therefore, at least 3 independent tests are necessary to determine the elastic constants of the DS alloy. Usually the tests to determine the three engineering elastic constants of $E_{0}, E_{90}$, and $G_{0}$ in Fig. 1 are adopted, because the test method and the deviation of the solidification direction have little effect on these engineering elastic constants. The relationship between these elastic constants and the elements of compliance matrix is described by Eq.(10).

$$
\overline{S_{33}}=1 / E_{0}, \quad \overline{S_{11}}=1 / E_{90}, \quad \overline{S_{55}}=1 / G_{0}
$$

Where, the elastic constants of $E_{0}$ and $E_{90}$ correspond to the Young's modulus in the direction of the blade height and blade chord, respectively, while $G_{0}$ corresponds to the shear rigidity around the $X_{3}$ axis as shown in Fig. 1 .

Next, consider the case where the alloy axis makes a difference from the structural axis due to manufacturing error as shown in Fig.2. In this case, the compliance matrix $\left[S^{\prime}\right]$ in the $x_{1} x_{2} x_{3}$ coordinate can be obtained by Eq.(11).

$$
\left[S^{\prime}\right]=[\Psi]^{T}[S][\Psi]
$$

Where, [Y] is stress transformation matrix from the $X_{1} X_{2} X_{3}$ coordinate to the $x_{1} x_{2} x_{3}$ coordinate, and superscript $T$ indicates the transpose of the matrix. Substituting Eq.(3) and Eq.(5) into Eq.(11), elastic constants of the actual DS blade, in which the direction between the alloy axis and the blade axis is slightly different, can be calculated. In addition, carrying out vibration analysis based on FEA by use of the compliance matrix of Eq.(11), the vibration characteristics of the actual DS blade can be obtained. In the most parts of the analysis, the deviation angle $\theta$ is changed within $\pm 15 \mathrm{deg}$, because the deviation angle of the actual DS blade is controlled within these angles. 


\subsection{Reuss Average and Voigt Average of Compliance}

It is reported that if the volume and the rotation angle in the transverse plane of the columnar crystal are known, the vibration analysis of the DS blade can be carried out with the practical accuracy by use of the averaging elastic constants by the Reuss and Voigt average ${ }^{(5)}$. However, it is difficult to get such data of the actual DS blade in advance. Therefore, in the actual mechanical design of the DS blade, the vibration analysis is carried out, assuming that the number of the columnar grains included in the blade is large enough, and the DS blade can be considered transverse isotropic. The Wells' method is one of the typical methods which can average the material constants over the transverse plane, assuming that the number of the columnar grains is infinite. Using Wells' method (Eq.(8) and Eq.(9)), the material constants are treated isotropically in the transverse plane. In this paper, one of the objectives is to examine the validity of the application of Wells' method for the actual DS blade, where the number of the columnar grains included in the blade is 5 to 10 at most.

In order to achieve this objective, first, the material constants obtained from the Wells' method are compared with those obtained by the Reuss method and the Voigt method, where the columnar grains of the finite number are averaged with respect to the volume. The Reuss average corresponds to the stress constant model, while the Voigt average the strain constant model $^{(5)}$. The compliances matrix by the Reuss and Voigt averages, $[S]_{R}$ and $[S]_{V}$, are equated as:

$$
\begin{aligned}
& {[S]_{R}=\sum_{k} V_{k}[\Psi]_{k}^{T}[S]_{k}[\Psi]_{k}} \\
& {[S]_{v}=\sum_{k} V_{k}\left([\Phi]_{k}^{T}[C]_{k}[\Phi]_{k}\right)^{-1}}
\end{aligned}
$$

Where, $V_{k}$ is the volume percent of each columnar grain. $[S]_{k}$ and $[C]_{k}$ are the elastic compliance and stiffness matrices of each columnar grain. $[\Psi]_{k}$ and $[\Phi]_{k}$ are the transformation matrices of stress and strain from the alloy coordinate to the structural coordinate.

Figure 3 shows the procedure for verifying the validity of the material constants obtained by the Wells' method, based on the Monte Carlo simulation. In this procedure, first, the number of the SC columnar grains included in the DS alloy is given. Next, after the rotation angle of each columnar grain in the transverse plane is selected at random, the DS alloy is assembled. The material constants of the DS alloy is calculated by the Reuss and Voigt methods. This process is repeated many times (one hundred times), and the calculated results are treated statistically to estimate the distribution of the elastic constants. The results are compared with the material constants calculated by the Wells' method based on the assumption of the isotropy in the transverse plane.

In the second verification procedure, the DS blade is modeled as a plate including 5 columnar grains as shown in Fig. 4. FE models, where the rotation angle $\phi$ of each columnar grain is changed in many patterns, are made, and the vibration analysis is carried out directly by use of FE models. This model is called the direct FE model hereafter. In this study, the results calculated by the direct FE model are considered correct, and are used as reference, because the columnar grains included in the DS blade are modeled exactly in the direct FE model. The distribution of the natural frequencies calculated by the direct FE models are compared with those calculated by FEA, which uses the material constant calculated by the Wells' method. Comparing the natural frequencies by both methods, prediction error of the natural frequency of the Wells' method for each vibration modes is clarified. In addition, the resonant stresses calculated by the direct FE model are compared to those calculated by the Wells' method to verify the prediction error. 


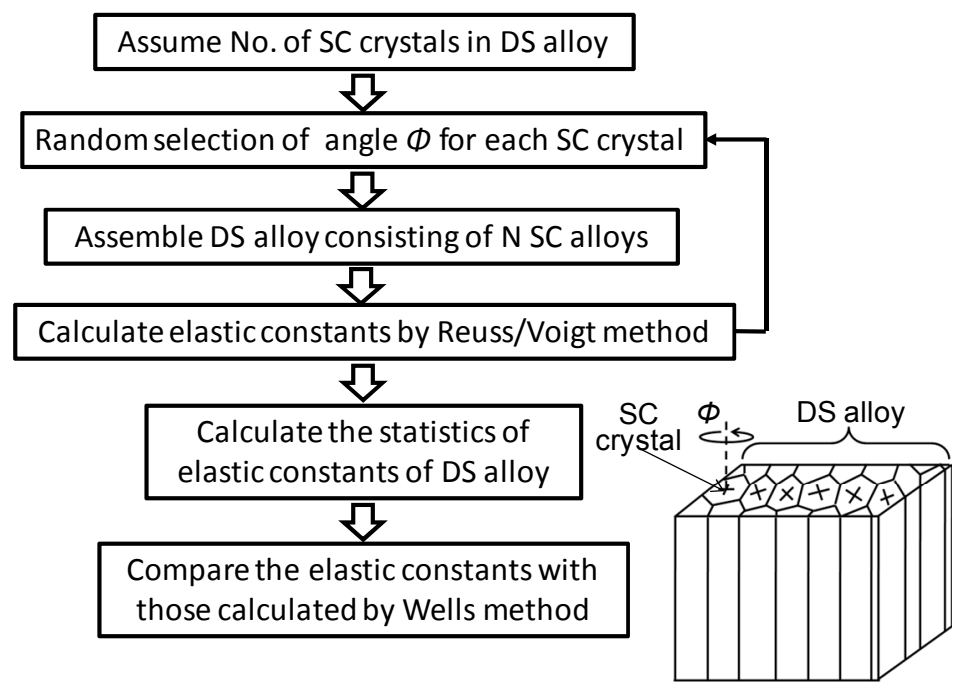

Fig. 3 Procedure of Monte Carlo simulation for elastic constants od DS alloy
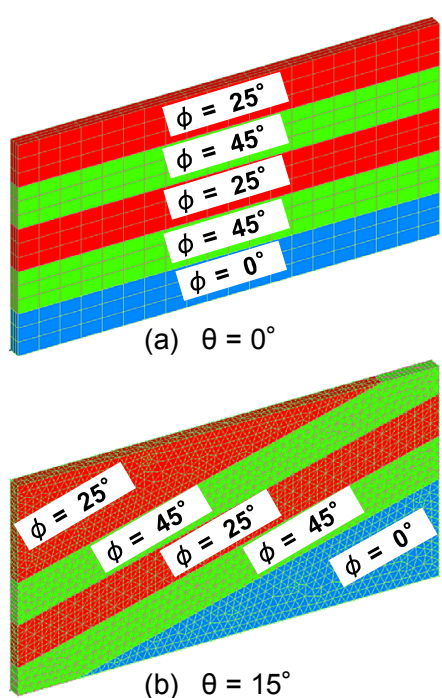

Fig. 4 DS blade model consisting of 5 SC alloys 


\section{Analysis Result}

\subsection{Effect of Deviation of Crystal Direction on Natural Frequency of SC Blade}

Since the DS alloy is a bundle of the SC alloys, it is important to examine the vibration characteristics of the SC blade for understanding the vibration characteristics of the DS blade. Therefore, the SC blade is modeled as a plate as shown in Fig. 2, and the effect of the crystal direction on the natural frequency was examined in detail. The SC blade has dimensions of $100 \times 200 \times 6$ in millimeter. The value of the compliances of the nickel single crystal $^{(3)}\left(\mathrm{s}_{11}=0.73 \times 10^{-5} \mathrm{MPa}^{-1}, \mathrm{~s}_{12}=-0.27 \times 10^{-5} \mathrm{MPa}^{-1}, \mathrm{~s}_{44}=0.80 \times 10^{-5} \mathrm{MPa}^{-1}\right)$ were used in the vibration analysis done by the commercial FEA code NASTRAN.

Figure 5 shows relationship between the natural frequency of the SC blade and the crystal direction. Figure 5(a) shows the frequency change due to the deviation of the crystal direction $\theta$, while Fig. 5 (b) shows the frequency change due to the deviations of the crystal directions $\phi$. The angels of $\theta$ and $\phi$ are defined as the rotation angle around [010] and [001] axis, respectively as shown in Fig. 2, and are changed in Fig. 5 from $0^{\circ}$ to $45^{\circ}$ considering the symmetry of the crystal.

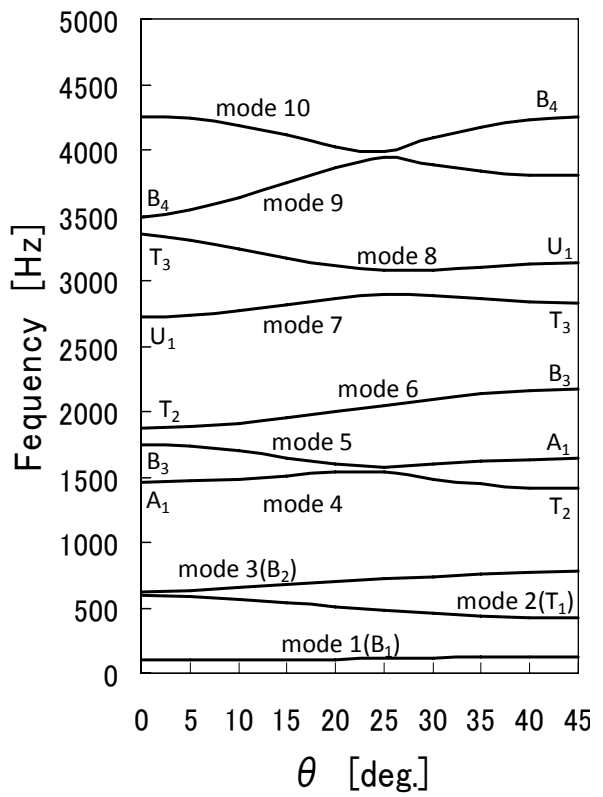

(a) Frequency change due to $\theta(\phi=0)$

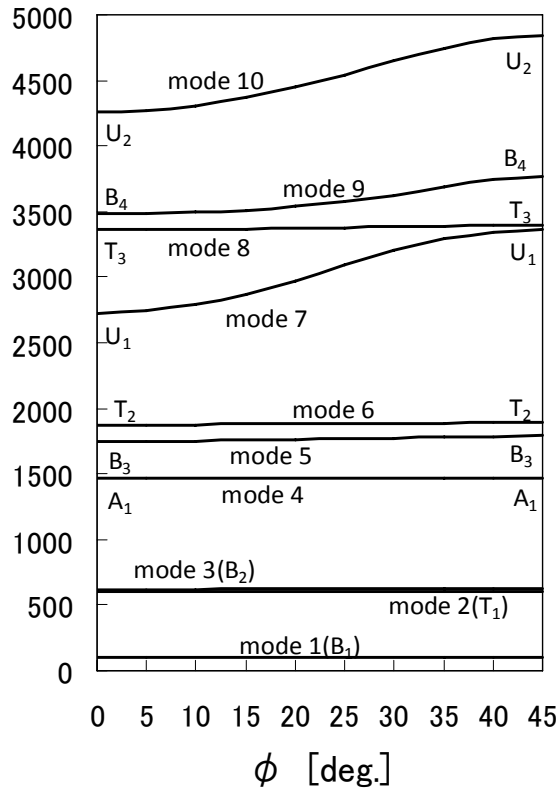

(b) Frequency change due to $\phi(\theta=0)$

Fig. 5 Natural frequency and crystal angle of SC blade

In Fig. 5 , the $\mathrm{B}_{1}, \mathrm{~B}_{2}, \mathrm{~B}_{3}$, and $\mathrm{B}_{4}$ mode denote the bending modes (out-of-plane modes) along the blade height, while the $A_{1}$ mode denotes the in-plane bending mode. On the other hand, $T_{1}, T_{2}$, and $T_{3}$ mode are torsion modes, while $U_{1}$ and $U_{2}$ mode are the bending modes along the blade chord.

Figure 6 shows the vibration modes of the SC blade, in which the solid lines correspond to the nodal lines. 


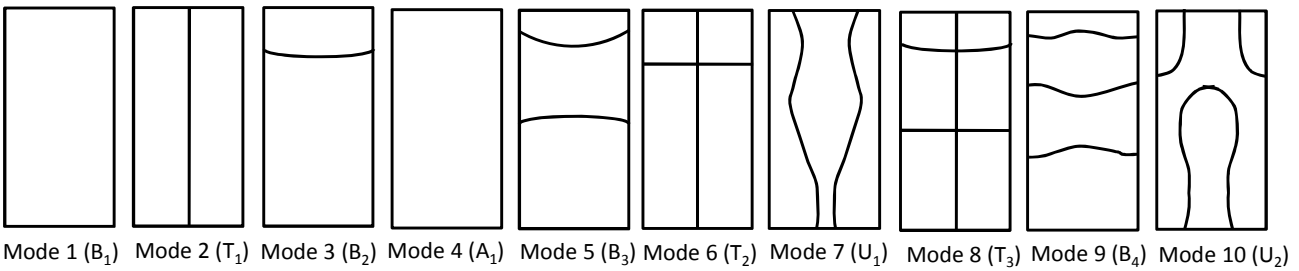

Fig. 6 Vibration mode of SC blade $(\theta=\phi=\gamma=0)$

Figure 7 denotes the effect of the deviation of the crystal direction on the material constants, and shows relationship between the material elastic constants $\left(E_{1}, E_{3}\right.$ and $\left.G_{3}\right)$ and the deviation of the crystal directions $(\theta, \phi)$. The material elastic constants of $E_{1}$ and $E_{3}$ correspond to Young's modulus along the blade chord and the blade height, respectively, while $G_{3}$ is the shear rigidity around $x_{3}$ axis, as shown in Fig. 2.

As shown in Fig. 5(b), the effects of the angle $\phi$ on the lower natural frequencies are very small, and the lower frequencies up to 6th mode are hardly changed due to the change of the angle $\phi$. Namely, it is clear that the effect of the angle $\phi$ is large only for the bending modes along the blade chord like the 7 th $\mathrm{U}_{1}$ mode and the 10 th $\mathrm{U}_{2}$ mode. On the other hand, as shown in Fig. 5(a), the effect of the deviation of the angle $\theta$ is observed from the lower vibration modes, and for the bending modes along the blade height (mode $\mathrm{B}_{1}$, mode $\mathrm{B}_{2}$, mode $A_{1}$, mode $B_{3}$ ), the natural frequencies increase with increase of the deviation of the angle $\theta$. Inversely for the torsion modes (mode $T_{1}$ and mode $T_{2}$ ), the natural frequencies decrease with increase of the deviation of the angle $\theta$.

The effects of the crystal direction on the natural frequencies shown in Fig. 5 can be clearly explained, comparing the results in Fig. 5, Fig. 7 and Fig. 8. Namely, as shown in Fig. 7(a), although Young's modulus along the blade height, $E_{3}$ hardly changes due to the deviation of the crystal angle $\phi, E_{3}$ increases with increase of the angle $\theta$, and reaches its maximum at $\theta=45^{\circ}$. On the other hand, as shown in Fig. 7(b), Young's modulus along the blade chord, $E_{1}$ increase with increase of the angle $\theta$ and $\phi$. And as shown in Fig. 7(c), although the shear rigidity, $G_{3}$ around $x_{3}$ axis hardly changes with increase of the angle $\phi, G_{3}$ decreases with increase of the angle $\theta$ and reaches its minimum at $\theta=45^{\circ}$. As a result, as shown in Fig. 5(a), with increase of the angle $\theta$, the natural frequencies of the bending modes along the blade height increase, while the natural frequencies of the torsion modes decrease. And the effect of the deviation of the angle $\phi$ appears only for the bending modes along the blade chord ( $\mathrm{U}_{1}$ and $\mathrm{U}_{2}$ modes), as shown in Fig. 5(b). 


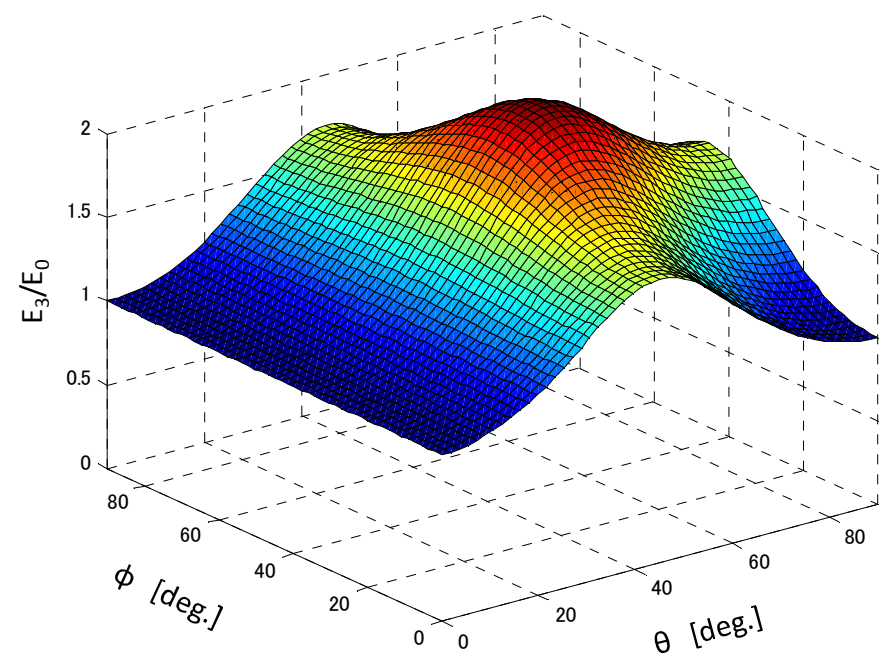

(a) Young's Modulus $E_{3}$

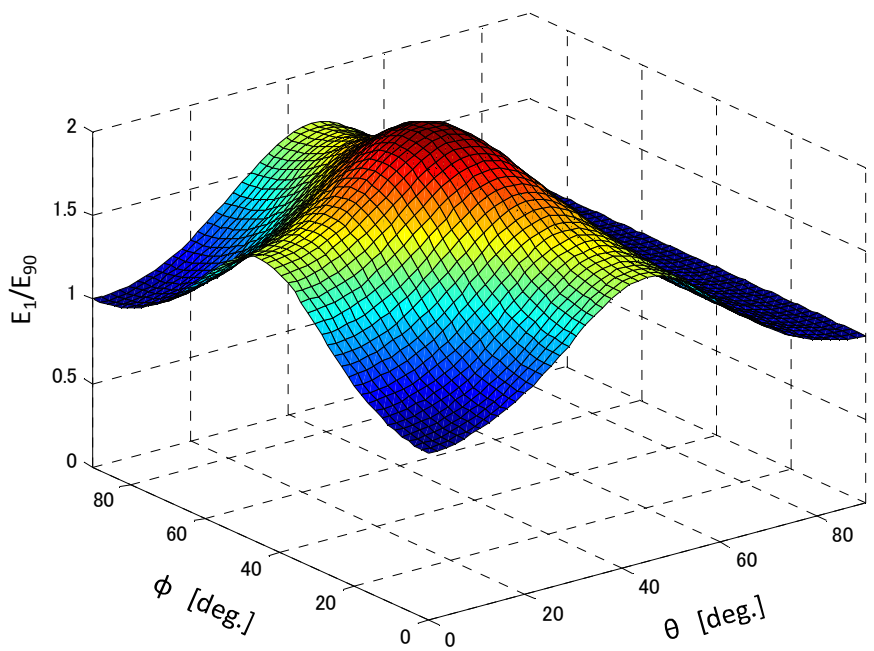

(b) Young's Modulus $E_{l}$

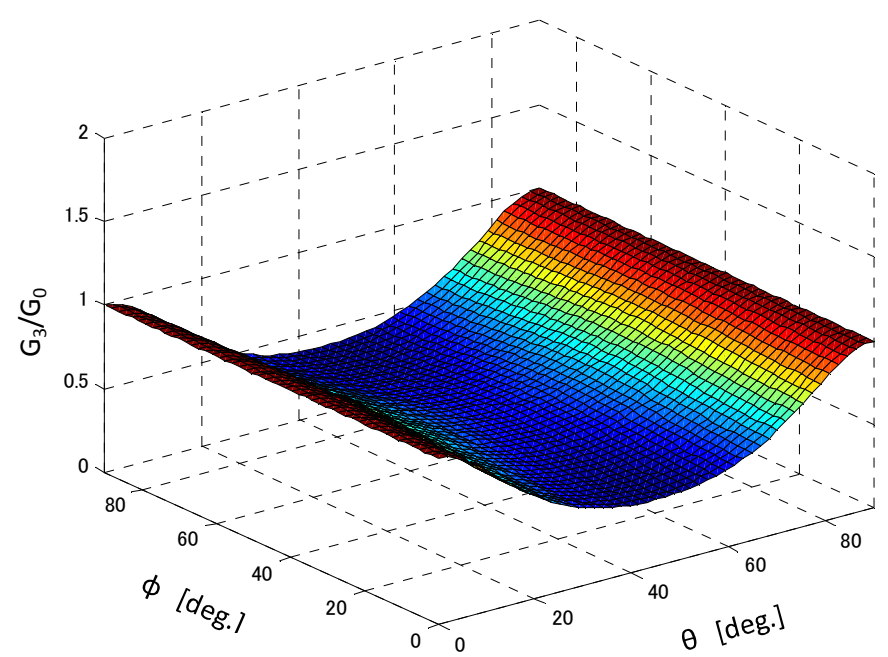

(c) Shear rigidity $G_{3}$

Fig. 7 Elastic constant and crystal angle $\theta, \phi$ of SC blade 


\subsection{Verification of Assumption of the Transverse Isotropy of DS Blade (Comparison of Elastic Constant)}

In order to examine whether the DS blade can be treated as a transversely isotropic material, the material constants averaged by the Wells' method are compared with those averaged by the Reuss method. When averaging the material constants by the Reuss method, the DS alloy is, first, assembled by selecting the crystal angle $\phi$ of each columnar grain at random, and then the material constants of the whole DS alloy is calculated by Eq.(12) as shown in Fig. 3. Because the elastic constants are calculated by averaging those of columnar grains by Eq.(12), the arrangement of the grains does not affect the results. This process is repeated one hundred times, and the calculated results are treated statistically to estimate the distribution of the elastic constants (Monte Carlo simulation).

Figure 8 shows the comparison of the elastic constants averaged by the Wells' method and the Reuss method. As shown in Fig. 8, even if the number of the columnar grains is small $(\mathrm{N}=5)$, the mean values of the elastic constants averaged by the Wells' method shows good agreement with those averaged by the Reuss method. The deviations of Young's modulus along the blade height, $E_{3}$ due to the deviation of the angle $\phi$ hardly appear when the angle $\theta$ is less than $20^{\circ}$ as shown in Fig. 8. This is because $E_{3}$ and $G_{3}$ of the single crystal alloy hardly change by the change of the angle $\phi$ when the angle $\theta$ is less than $20^{\circ}$, as shown in Fig. 7(a) and Fig. 7(c). On the other hand, as for the Young's modulus along the blade chord, $E_{1}$, the deviation caused by the randomness of the angle $\phi$ becomes large, when the number of the columnar grains is small.

Figure 9 shows the relationship between the number of the crystals and the variation (the ratio between the standard deviation and the mean value) of the elastic modulus under the assumption of $\theta=15^{\circ}$. As shown in Fig. 9, the variation of the elastic modulus decreases monotonically with the increase of the number of the crystals. It can be also said from Fig. 9 that in order to reduce the standard deviation of the Young's modulus along the blade chord, $E_{I}$ to less than $1 \%$, it is necessary to increase the number of columnar grains included in the DS alloy up to 200. In the actual blade design, however, only the natural frequencies of lower vibration modes, in which the effects of $E_{3}$ and $G_{3}$ are dominant, are adjusted. Therefore, it can be said that since the standard deviations of $E_{3}$ and $G_{3}$ are less than $1 \%$ even if the number of the columnar grains is small (around 5), the vibration analysis of the DS blade can be carried out under the assumption of the transverse isotropy.

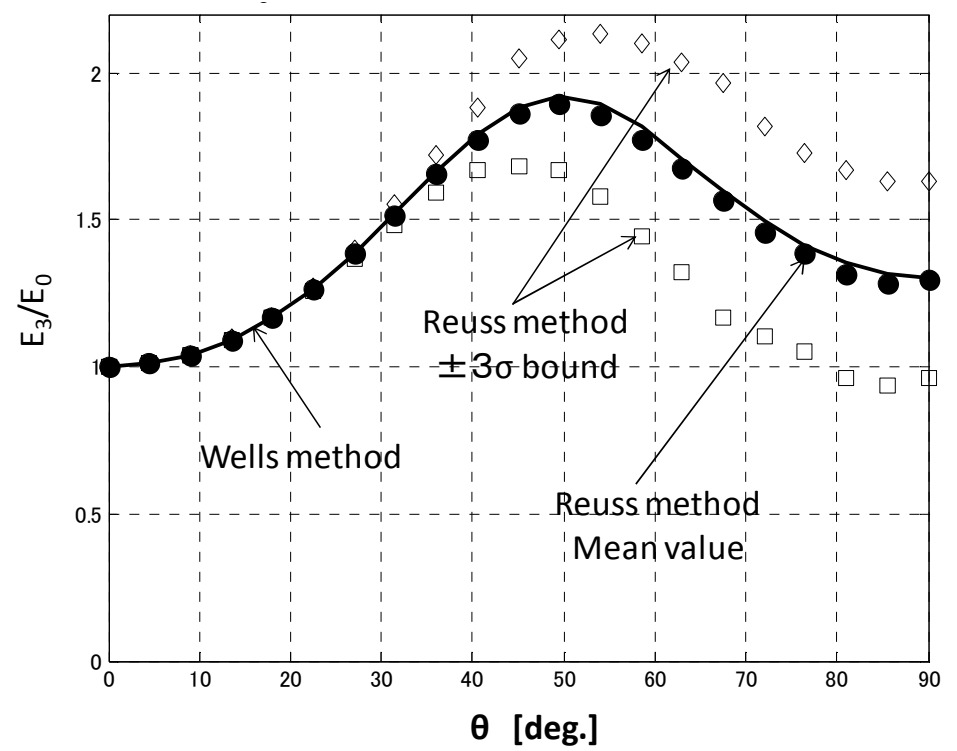

Fig. 8 Young's modulus $E_{3}$ by Wells and Reuss average method ( $\left.\mathrm{N}=5\right)$ 


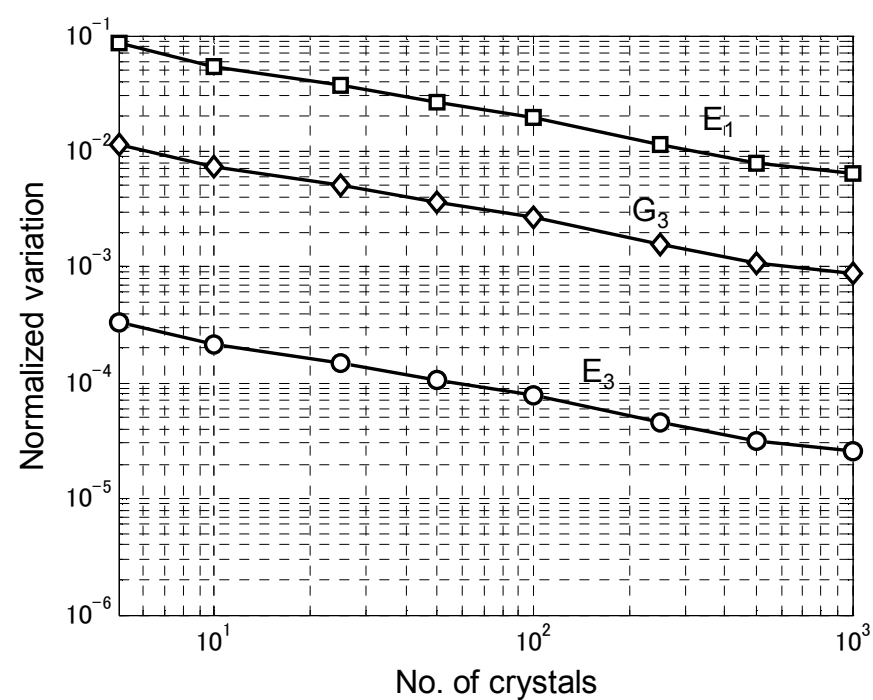

Fig. 9 No. of crystals and variation of elastic modulus by Reuss method $(\theta=15 \mathrm{deg})$

\subsection{Verification of Assumption of the Transverse Isotropy of DS Blade (Comparison of Natural Frequency)}

Figure 10 shows the comparison of the natural frequencies of the DS blade with $\theta=0^{\circ}$ in Fig. 4. The open circle denotes the natural frequency of the DS blade consisting of different combination of the columnar grains, where the five columnar grains are modeled exactly and the natural frequency is calculated by the direct FEA. The solid line corresponds to the natural frequency of the DS blade calculated by FEA, where the elastic constants averaged by the Wells' method are used as a material property of the DS blade with the transverse isotropy. In the vibration analysis, it is assumed that the DS blade consisting of five columnar grains has one columnar grain of $\phi=0^{\circ}$, two columnar grains of $\phi=25^{\circ}$, and two columnar grains of $\phi=45^{\circ}$, based on the reference ${ }^{(3)}$. Under this assumption, the number of all combinations of the columnar grains is sixteen, and therefore, the vibration analysis was carried out for all sixteen models as shown in Fig. 10.

Figure 11 shows the frequency error between the average of the direct FEA results of 16 models and the result by the Wells' method. In Fig. 11, the deviation of the natural frequencies of the 16 models, $3 \sigma / \mu$ ( $\sigma$ : standard deviation, $\mu$ : mean value) is also plotted. As shown in these figures, the natural frequencies of the lower bending and torsion modes calculated by both methods show good agreement, and the frequency error is less than $1 \%$. In addition, the frequency deviation of the 16 models is negligible for the lower bending and torsion modes. On the other hand, it is shown that for the higher mode of $U_{l}$ (the bending mode along the blade chord), the frequency error and frequency deviation become large. However, in the mechanical design of the DS blade, the natural frequency of the higher mode is out of frequency control. Therefore, it can be said from these results that even if the number of the columnar grains is small, the DS blade can be treated as a transversely isotropic material, and the vibration analysis can be carried out using the elastic constants averaged by the Wells' method.

Figure 12 shows the comparison of the natural frequencies of the DS blade with $\theta=15^{\circ}$. Figure 13 shows the frequency error between the average of the direct FEA results of 16 models $\left(\theta=15^{\circ}\right)$ and the result by the Wells' method. In Fig. 13, the deviation of the natural frequencies of the 16 models, $3 \sigma / \mu$ is also plotted. Even in case that the angle of $\theta$ becomes the allowable limit, the natural frequencies of the lower bending and torsion modes calculated by both methods show good agreement, and the frequency error is almost less than $1 \%$. The frequency deviation of the 16 models is also negligible for the lower bending and torsion modes. 


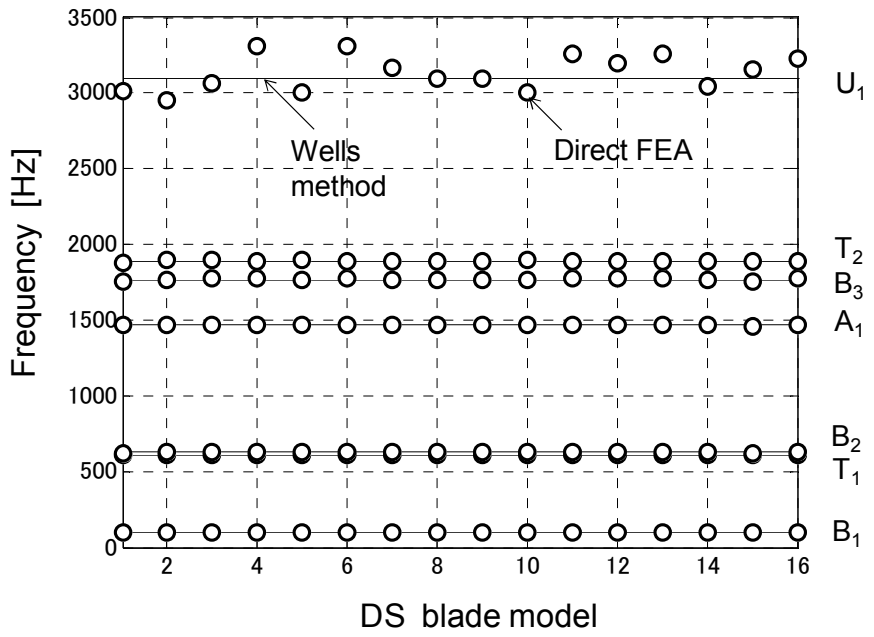

Fig. 10 Frequency of DS blade ( $\theta=0$ deg. $)$

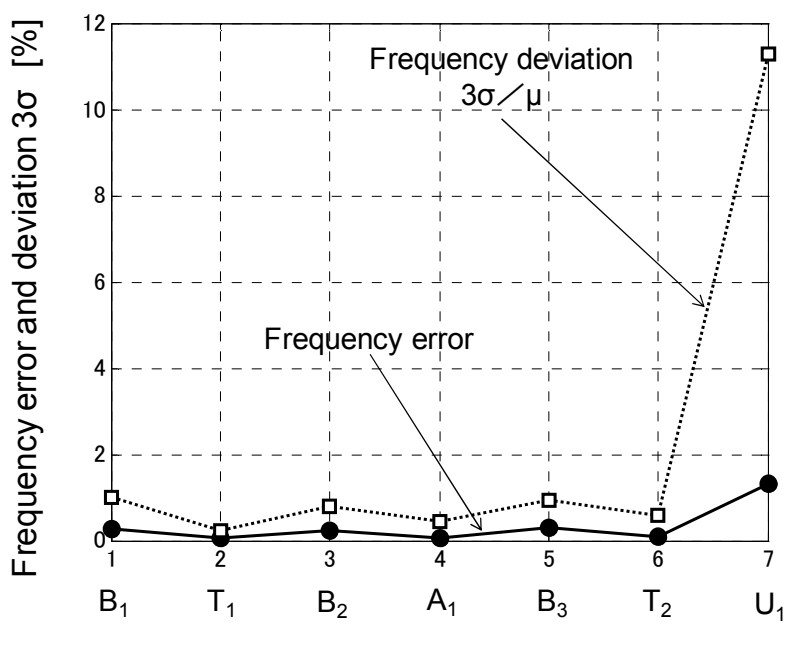

Mode No.

Fig. 11 Frequency error and deviation of DS blade ( $\theta=0$ deg.)

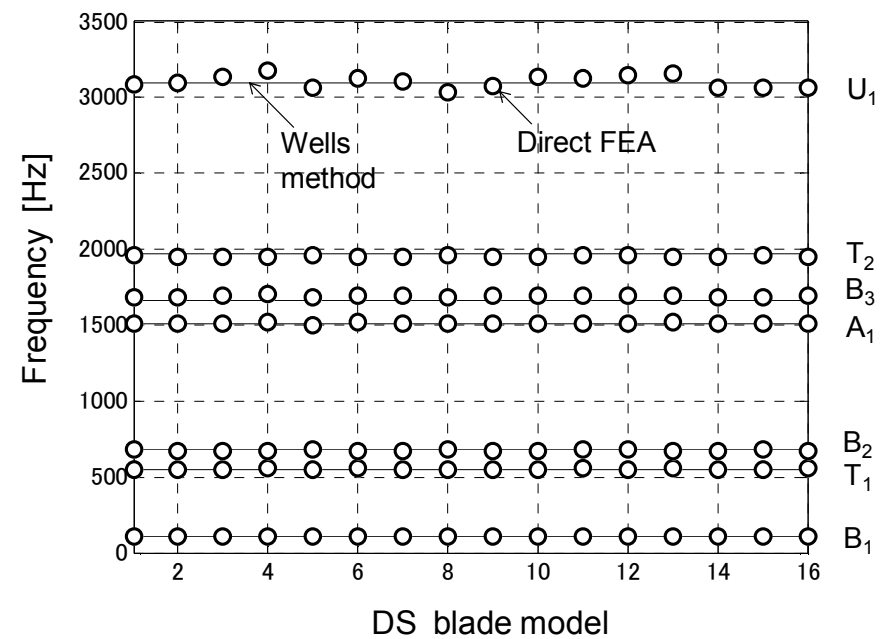

Fig. 12 Frequency of DS blade ( $\theta=15$ deg. $)$ 


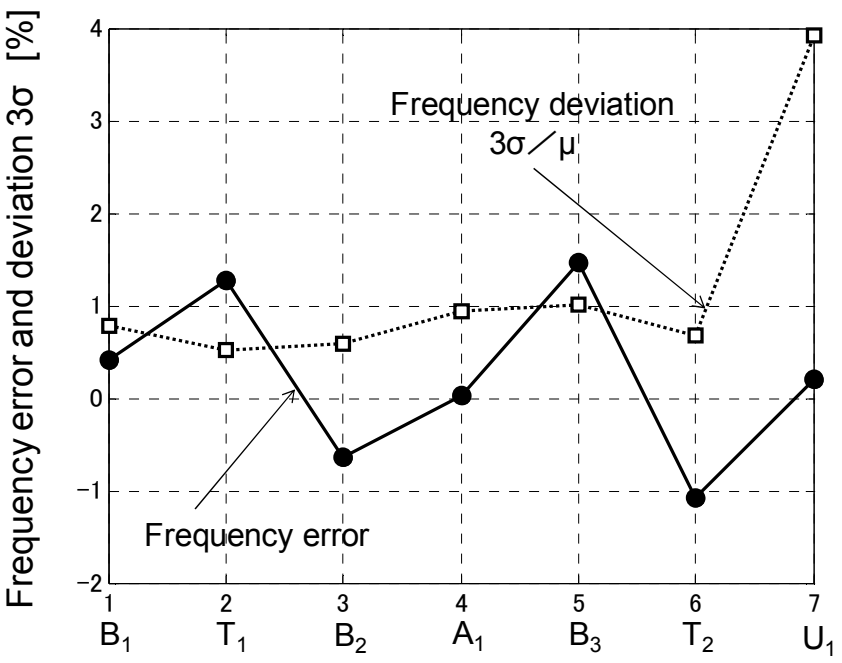

Mode No.

Fig. 13 Frequency error and deviation of DS blade $(\theta=15$ deg. $)$

Figure 14 shows the vibratory stress (Mieses stress) distribution of the 7th $\mathrm{U}_{1}$ mode, for which the prediction error of the natural frequency in Fig. 13 is maximum. In Fig. 14, the vibratory stress distribution calculated by the Wells' method based on the assumption of the transverse isotropy is compared with that calculated by the direct FEA. As shown in Fig. 14 , the vibratory stress modes changes remarkably if the direction of the crystal angle $\theta$ changes from $0^{\circ}$ to $15^{\circ}$. However, the difference between the vibratory stress distributions calculated by both methods is small for the same crystal angle $\theta$.

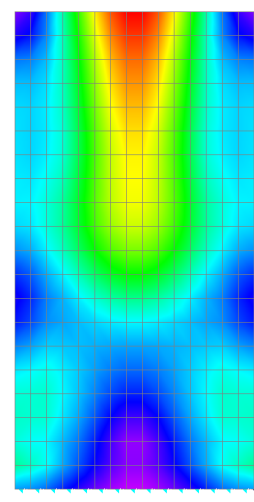

Wells average

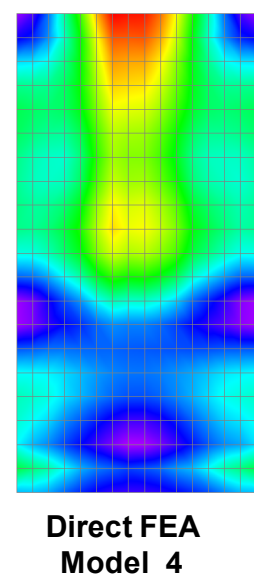

(a) $\theta=0$ deg.

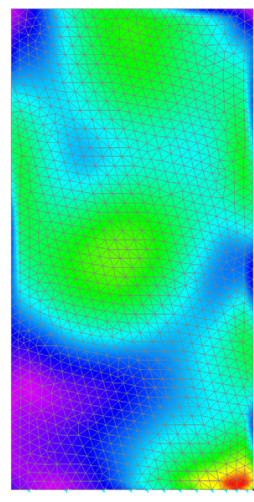

Wells average

(b) $\theta=15$ deg.

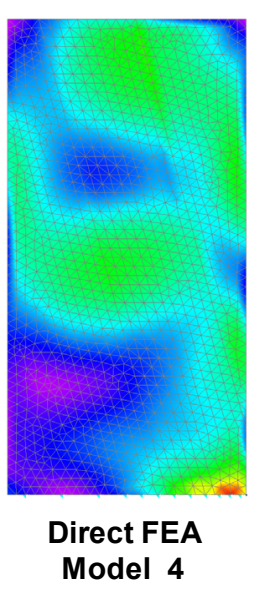

Min.

Fig. 14 Distribution of vibratory stress (Mieses stress, Mode 7)

Figure 15 shows the error of the maximum resonant stress between the average of the direct FEA results of 16 models and the result by the Wells' method. In Fig. 15, the deviation of the maximum resonant stress of the 16 models, $3 \sigma / \mu$ ( $\sigma$ : standard deviation, $\mu$ : mean value) is also plotted. In the analysis of resonant stress, the vibration mode is normalized so as that the modal mass is unit value, and the unit excitation force is applied on the grid point with the maximum displacement. In addition, the stress concentration factor and so on are not considered, because the purpose of Fig. 15 is the relative comparison between the vibratory stresses of the DS blade calculated by the direct FEA and 
Wells' method based on the assumption of the transvers isotropy. As shown in Fig. 15, the maximum resonant stress error of around $8 \%$ appears, while the maximum deviation $(3 \sigma / \mu)$ reaches around $16 \%$. It seems that because the vibratory stress is local physical value, its prediction error caused by the assumption of the transverse isotropy becomes large as well as its deviation, comparing to those of the natural frequency in Fig.11. However, on the mechanical design of the DS blade, it is possible to weave the safety factor into the predicted vibratory stress, if these probable prediction errors are known in advance.

Figure 16 shows the error of the maximum resonant stress between the average of the direct FEA results of 16 models $\left(\theta=15^{\circ}\right)$ and the result by the Wells' method. Comparing Fig. 15 and Fig. 16, it is shown that the deviation of the resonant stress $(3 \sigma / \mu)$ increases slightly. However, because the maximum deviation is around $20 \%$ at most, it is concluded that the assumption of the transverse isotropy can be applied to the vibration analysis of the actual DS blade.

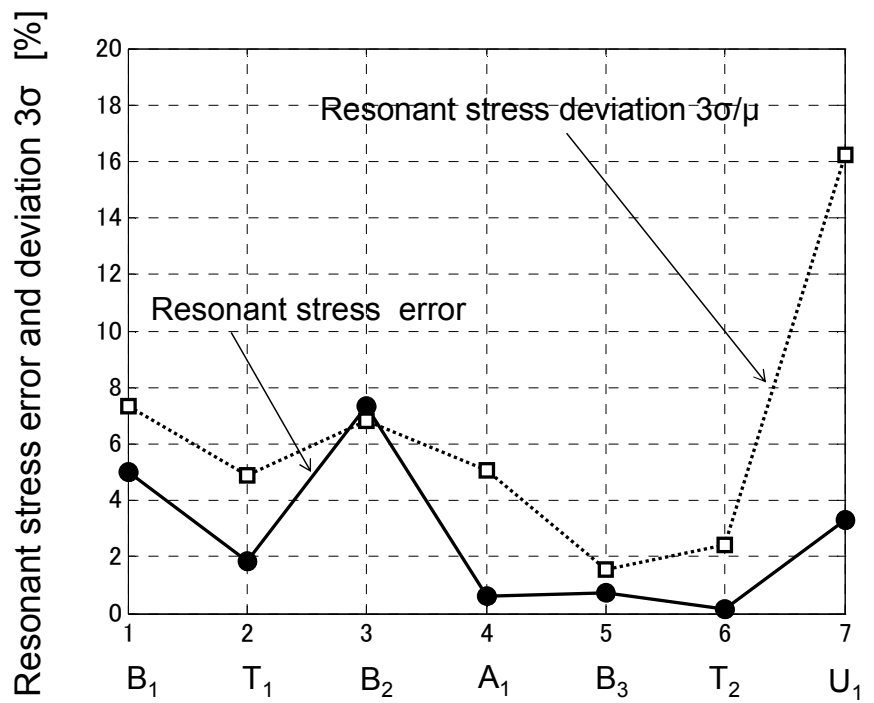

Mode No.

Fig. 15 Resonant stress error and deviation of DS blade $(\theta=0$ deg. $)$
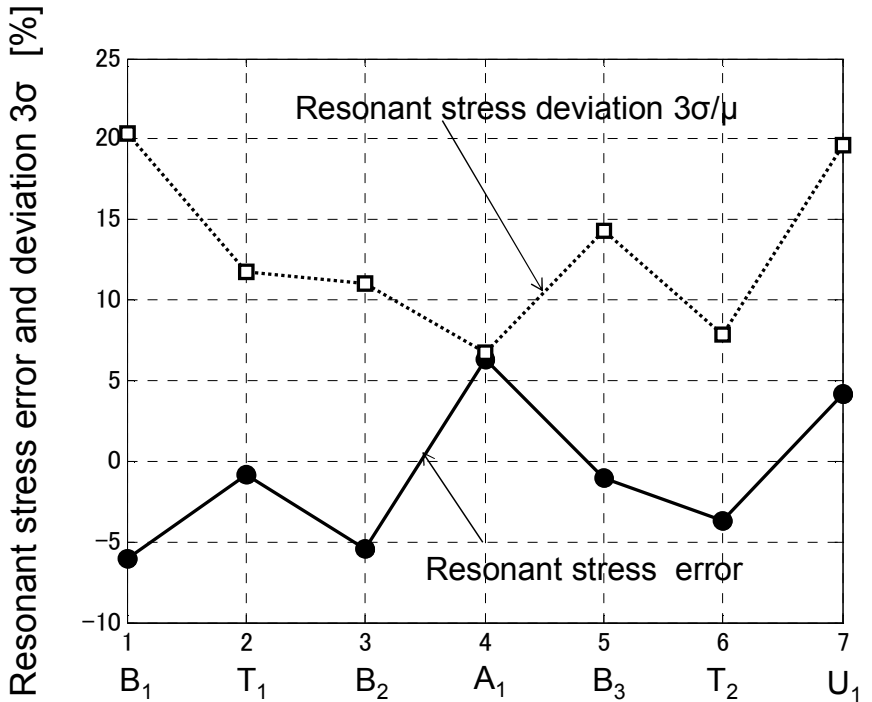

Mode No.

Fig. 16 Resonant stress error and deviation of DS blade $\quad(\theta=15$ deg. $)$ 


\section{Conclusions}

In this study, first, the effect of the anisotropy of elastic constants on the vibration characteristics of the SC and DS blade was investigated systematically. Second, the validity of the assumption of the transverse isotropy for the DS blade was examined, carrying out the Monte Carlo simulation on the elastic constants and FE analysis of the DS blade. From these results, it is clarified that the frequency change of SC blade caused by the deviation of the crystal direction corresponds to the change of the elastic modulus for all vibration modes, including the bending mode along the blade height, the bending mode along the blade chord, and the torsion modes. It is also said that even if the number of the columnar grains included in the DS blade is small, the prediction error of the natural frequency caused by the assumption of the transverse isotropy is negligible small, and the prediction error of the resonant stress is also an allowable level. Therefore, it is concluded that the DS blade can be treated as a transversely isotropic material, and the vibration analysis can be carried out using the elastic constants averaged by the Wells' method.

\section{References}

(1) Kaneko, H., and Sakon, T., A Study on Biaxial Thermal Fatigue Life of A Directionally Solidified Alloy for Gas Turbine Blade, Proceedings of International Conference on Power Engineering-97, Vol.2(1997), pp.113-117.

(2) Manetti, M., Giovannetti, L., Pieroni, N., Horculescu, H., Peano, G., Zonfrillo, G. and Giannozzi, M., The Dynamic Influence of Crystal Orientation on a Second Generation Single Crystal Material for Turbine Buckets, Proceedings of ASME Turbo Expo 2009, GT2009-59091(2009), pp.1-9.

(3) T. Hasebe, M. Sakane and M. Ohnami, High Temperature Low Cycle Fatigue and Cyclic Constitutive Relation of MAR-M247 Directionally Solidified Superalloy, Trans. of the ASME, Journal of Engineering Material and Technology, Vol. 114(1992), pp162-167.

(4) Hasebe, T. and Sakane, M., Vibration of Nickel-Base Single Crystal and Directionally Solidified Superalloy Plates, JSME International Journal, Series A, Vol. 37, No. 4(1994), pp.481-487.

(5) Hasebe, T., Sakane, M. and Ohnami, M., Elastic Anisotropy of Directionally Solidified Superalloy, Trans. of the ASME, Journal of Engineering Material and Technology, Vol. 114(1992), pp141-146.

(6) Wells., C. H., The Elastic Constants of a Directionally-Solidified, Nickel-Base Superalloy, Mar. M-200, Trans. of the ASM, Vol. 60(1967), pp270-271. 